\title{
UEBER DIE SELBSTAUFLADUNG KORPUSKULAR STRAHLENDER KOERPER.
}

\author{
VON E. SCHWEIDLER.
}

[Summary.-1. The observed phenomena, which have led to hypotheses regarding the existence of corpuscular cosmic rays, are briefly discussed and the importance is pointed out of deducing the theoretical consequences resulting from the charged state of the emitting cosmic bodies. 2. The characteristics of corpuscular rays are discussed, especially the "radiation potential," $P$ (quotient of the kinetic energy and the electric charge of a particle), and the magnetic deflectibility. 3. The stationary charge of a sphere, which spontaneously emits corpuscular rays into a surrounding vacuum, is computed; it is larger than the product $\mathrm{Pa}$ ("radiation potential" times radius of sphere), and increases with intensity of emission; the emitted particles are compelled to turn back at a distance $A$, the values of which are computed; numerical examples for the Moon, as the supposed source of radiation, are added. 4 . The stationary charge during simultaneous spontaneous emission of positive and negative corpuscular rays is computed. 5. Analogous calculations are made on the assumption that a sphere with a strongly ionized surface emits a spontaneous radiation and hence on account of its charging, a compensating field-driven ion-radiation is emitted; in an equilibrium condition, therefore, at a great distance, there exist both positive and negative rays of equal average intensity and equal velocity; the results are applied to the case of the Sun and several numerical examples are given.]

\section{EINLEITUNG.}

Strahlungen korpuskularer Natur spielen in der kosmischen Physik eine wichtige Rolle. Zunächst sind es die Nordlichterscheinungen, die nach der von Birkeland aufgestellten und insbesondere von Störmer; Lenard und Vegard weiter entwickelten Theorie auf solche Strahlen zurückgeführt werden; ihre quantitativen Merkmale (Ladung, Masse, Geschwindigkeit) sind allerdings bisher nicht mit voller Sicherkeit anzugeben. Während aus der Lage des Gürtels grösster Nordlichthäufigkeit auf Strahlen sehr grosser magnetischer Steifigkeit (also entweder sehr schnelle $\beta$-Strahlen oder Strahlen grosser Masse) zu schliessen wäre, deuten die beobachteten räumlichen Verhältnisse (Höhe und vertikale Ausdehnung der Lichterscheinungen) unter Berücksichtigung der Bahnform und der Absorption auf negative Strahlen verhältnismässig geringer Steifigkeit. ${ }^{1}$ Diesen Widerspruch sucht bekanntlich Störmer durch die Annahme zu lösen, dass ausserterrestrische ringförmige Ströme-ebenfalls korpuskularer Natur-

‘C. Störamer, Geofys. Publ. 1, Nr. 5, Kristiania, 1921. 
schon weit ausserhalb der Atmosphäre die Bahnen der ankommenden Teilchen durch ihr magnetisches Feld beeinflussen. Auch die Wirkung der elektrostatischen Abstossungskräfte zwischen den Teilchen eines Schwarmes ist theoretisch noch nicht ganz befriedigend aufgeklärt.

Neben den Nordlichten sind aber noch andere Erscheinungen bekannt, die auf die Existenz kosmischer Korpuskularstrahlen hinweisen. Zunächst ist es die von Gockel und von Hess zuerst beobachtete, in höheren Schichten der Atmosphäre ionisierend wirksame sehr durchdringende Strahlung, die wenigstẹns indirekt (als von ihnen erzeugte Sekundärstrahlung) auf von aussen kommende Korpuskularstrahlen zurückgeführt werden könnte. Ferner ist aus der Aufrechterhaltung der negativen Erdladung auf eine von aussen kommende, sehr durchdringende negative Korpuskularstrahlung geschlossen worden (Simpson, ${ }^{2}$ Swann, ${ }^{3}$ Schweidler ${ }^{4}$ Seeliger. ${ }^{5}$ )

Endlich hat Bauer ${ }^{6}$ aus erdmagnetischen Daten die Existenz eines Stromsystems festgestellt, dessen Stromlinien im allgemeinen in den Polarkappen der Erde aus ihrer Oberfläche austreten und im Aequatorialgürtel eintreten. Auch er hält eine Erklärung durch Korpuskularstrahlen für möglich, die dann in niedrigen und mittleren Breiten positive, in hohen Breiten negative Ladungen durch die Erdatmosphäre hindurch transportieren; eventuell wären auch Strahlen entgegengesetzter Ladung und Richtung (also von der Erde ausgehend) denkbar. In jedem Falle wäre ihnen eine ausserordentlich grosse Durchdringungsfähigkeit zuzuschreiben.

Bei allen diesen auf mehr oder minder ausreichenden Beobachtungstatsachen beruhenden und daher in verschiedenem Grade präzisierten Hypothesen erscheint es nun von Interesse, die Konsequenzen zu betrachten, zu denen man in Bezug auf den Ladungszustand der die Strahlen aussendenden Weltkörper gelangt.

2. DIE CHARAKTERISTISCHEN KONSTANTEN KORPUSKULARER STRAHLEN.

Eine sogennante "homogene" Korpuskularstrahlung ist eindeutig bestimmt durch die Zahl, Masse, Ladung, und Geschwindigkeit der Teilchen. Wir bezeichnen die in der Zeiteinheit (sec) die

1G. C. Smason, Nainte, 69, 270, 1904.

IW. F. G. SWanN, Terr. Mag. 20, 105, 1915.

'E. v. SchweIDLER, Wien Ber. 127, 515, 1918, und Ann. d. Phys. (4) 63, 726, 1920.

'R. SeEliger, Ann. d. Phys. (4) 62, 446, 1920.

IL. A. BAUER, Terr. Mag. 25, 145, 1920. 
Flächeneinheit $\left(\mathrm{cm}^{2}\right)$ des Querschnittes eines Bündels passierende Teilchenzahl mit z; die Ladung (in statischen Einheiten) mit $e$; die Masse (in $g$ ) oder genauer-in Berücksichtigung der Abhängigkeit der Masse von der Geschwindigkeit-die Ruhmasse mit m; endlich die Geschwindigkeit mit $v=\beta . c$, wobei $c$ die Lichtgeschwindigkeit und $\beta$ ein zwischen 0 und 1 liegender dimensionsloser Faktor ist.

Für die bei der Lorentz-Transformation auftretende Grösse $\frac{1}{\sqrt{1-\beta^{2}}}$, führen wir die abkürzende Bezeichnung $\eta$ ein; die kinetische Energie eines Teilchens ist dann nach den Formeln der speziellen Relativitätstheorie:

$$
E=m c^{2}\left(\frac{1}{\sqrt{1-\beta^{2}}}-1\right)=m c^{2}(\eta-1)
$$

was bekanntlich bei kleinen Geschwindigkeiten $(\beta<<1)$ in die gewöhnliche Formel $E=\frac{1}{2} m c^{2} \beta^{2}$ übergeht.

Drücken wir $E$ als Produkt $P e$ einer Spannung $P$ und der Teilchenladung $e$ aus, so bedeutet $P$ diejenige Potentialdifferen $z$ (in stat. Einh.), die das ursprünglich ruhende Teilchen in einem beschleunigenden Felde durchlaufen muss, um die Endgeschwindigkeit $\beta c$ zu erhalten, bezw. die Potentialdifferenz, die bei verzögerter $\mathrm{Be}-$ wegung das Teilchen von der Anfangsgeschwindigkeit $\beta c$, auf die Geschwindigkeit Null abbremst. Wir wollen $P$, das (in Volt gemessen) gewöhnlich die "Voltgeschwindigkeit" genannt wird, hier kurz das "Strahlpolential" nennen und durch $P=\frac{m c^{2}}{e}(\eta-1)$ darstellen. Durchläuft ein Teilchen mit dem Strahlpotential $P_{0}$ eine beschleunigende oder bremsende Potentialdifferenz $\Pi$, so ist am Ende der Bahn sein Strahlpotential $P=P_{\circ} \pm \Pi$. Für die Berechnung der Zeit, die es zu dieser Strecke braucht, ist es von Bedeutung, dass trotz Aenderung der kinetischen Energie die Geschwindigkeit sich nur wenig ändert, falls $\beta_{0}$ sehr gross ist. Durchläuft z. B. ein Teilchen mit $\beta_{0}<0.3$ in einem bremsenden Feld konstanter Stärke bis zum Umkehrpunkt die Strecke $l$, so ist die Bewegung sehr annähernd eine gleichförmig verzögerte und daher die mittlere Geschwindigkeit $\bar{v}=\frac{1}{2} \beta_{0} c$. Ist dagegen z. B. $\beta_{\circ}=$ 0.9999 und $l$ bei entsprechend verstärktem Felde ebenso gross wie früher, so ist nach Durchlaufen von 0.98 l die Geschwindigkeit immer noch grösser als $0.9 c$, die Bremsung erfolgt rapid im letzten 
kleinen Stück der Bahn und die mittlere Geschwindigkeit liegt nur wenige Prozente unter der Anfangsgeschwindigkeit.

Das für das Verhalten in magnetischen Feldern charakteristische Produkt $H \rho$ (in $\Gamma$. cm) aus magnetischer Feldstärke und Krümmungsradius ist in unserer Bezeichnungsweise:

$$
H \rho=\frac{m c^{2}}{e} \cdot \eta \beta
$$

denn $m \eta$ ist die "transversale Masse" und $\beta c$ die Geschwindigkeit. Für sehr grosse Geschwindigkeiten ( $\beta$ nahe gleich 1 , $\eta$ gross gegen 1) werden daher die Grössen $H \rho$ und $P$ (in stat. Einh.) einander nahezu gleich. Die numerischen Werte veranschaulicht die folgende Tabelle:

\begin{tabular}{c|c|c|c}
\hline$\beta$ & $\eta$ & $(\eta-1)$ & $\eta \beta$ \\
\hline 0.0 & 1.000 & 0.000 & 0.000 \\
0.1 & 1.005 & 0.005 & 0.100 \\
0.2 & 1.020 & 0.020 & 0.204 \\
0.3 & 1.048 & 0.048 & 0.314 \\
0.4 & 1.091 & 0.091 & 0.436 \\
0.5 & 1.155 & 0.155 & 0.578 \\
0.6 & 1.250 & 0.250 & 0.750 \\
0.7 & 1.400 & 0.400 & 0.980 \\
0.8 & 1.667 & 0.667 & 1.334 \\
0.9 & 2.294 & 1.294 & 2.065 \\
0.95 & 3.203 & 2.203 & 3.043 \\
0.99 & 7.089 & 6.089 & 7.018 \\
0.995 & 10.01 & 9.012 & 9.962 \\
0.999 & 22.36 & 21.36 & 22.34 \\
$1-10-4$ & 70.71 & 69.71 & 70.70 \\
$1-10-5$ & 223.6 & 222.6 & 223.6 \\
$1-10-6$ & 707.1 & 706.1 & 707.1 \\
\hline
\end{tabular}

Für die Berechnung von $P=\frac{m c^{2}}{e}(\eta-1)$

$$
\text { und } H \rho=\frac{m c^{2}}{e} \eta \beta
$$

gelten die Werte:

$$
\begin{aligned}
\frac{m c^{2}}{e} & =1694 \text { bei Elektronen } \\
& =3.13 \times 10^{6} \text { bei } H \text {-Kernen } \\
& =6.21 \times 10^{8} \text { bei } a \text {-Teilchen }
\end{aligned}
$$

und leicht analog zu ermittelnde Werte für positive Atomionen anderer Art. 
3. DIE SELBSTAUFLADUNG DURCH SPONTANE KORPUSKU ${ }^{*}$ IRSTRAHLUNG.

Wir nehmen an, dass eine feste Kugel vom Radius $a$ von absolutem Vakuum ungeben sei und spontan, d. h. ohne Mitwirkung eines elektrischen Feldes, nach Art eines radioaktiven Körpers eine homogene Korpuskularstrahlung (Strahlpotential $P_{0}$ ) aussende. Es sei $z$ die Zahl der Teilchen pro Flächen - und Zeiteinheit, also $I=$ $4 \pi a^{2} z e$ der ganze ausgesandte Strom. Der Einfachheit halber setzen wir weiter voraus, dass die Strahlung die Oberfläche überall senkrecht verlasse.

Die Kugel nimmt dann eine Ladung entgegengesetzten Vorzeichens an, erzeugt dadurch ein bremsendes Feld und erreicht asymptotisch einen stationären Ladungszustand, bei dem in der Zeiteinheit ebensoviele Teilchen auf die Kugel zurückgetrieben als spontan emittiert werden. Es sei Emission negativer Teilchen und daher positive Aufladung vorausgesetzt; der umgekehrte Fall ist dann natürlich durch Vorzeichenswechsel in den Formeln erledigt. Zunächst-vom ungeladenen Zustand beginnend-gehen die emittierten Teilchen in unendliche Entfernung, solange bis die Kugel eine Ladung im Betrage $P_{0} a$ angenommen hat; sobald dieser Grenzwert überschritten ist, werden alle weiterhin ermittierten Teilchen in endlicher Entfernung $A$ zur Umkehr gebracht und gelangen nach einer gewissen Zeit $2 T$ (Steigdauer und Falldauer) wieder zurück. Im stationären Zustande ist die Ladung der Kugel $Q_{a}>P_{\diamond} a$, die zwischen $r=a$ und $r=A$ auf dem Hin-oder Rückwege befindilchen Teilchen haben eine Gesamtladung $-Q^{\prime}$ und es muss die Bedingung erfüllt sein:

Andererseits muss gelten:

$$
Q_{a}-Q^{\prime}=P_{0} a
$$

$$
Q^{\prime}=4 \pi a^{2} z e .2 T=I .2 T
$$

da eben die negative "Teilchenatmosphäre" aus den innerhalb der Zeit $2 T$ emittierten Teilchen besteht.

Die stationäre Ladung $Q_{a}$, die Umkehrentfernung $A$ und die Steigdauer $T$ sind also abhängig von $P_{0}, a$ und $I$. Die Berechnung dieser Grössen und der Feldverteilung im allgemeinen Falle ist ziemlich kompliziert; es seien daher hier nur einige Spezialfälle bahandelt.

Es werde angenommen, dass die Anfangsgeschwindigkeit $\beta_{0}$, klein und daher die Teilchenmasse praktisch konstant sei; ferner dass die Emission $I$ so klein sei, dass das Feld der Raumladung $-Q^{\prime}$ vernachlässigt werden kann. Dann lässt sich zu jedem vorge- 
gebenen Werte der Umkehrentfernung $A$ die zugehörige Ladung $Q_{a}$ und die Steigdauer $T$ berechnen. Zunächst folgt aus den Potentialwerten $V_{a}=\frac{Q_{a}}{a}$ und $V_{A}=\frac{Q_{a}}{A}=\mathrm{V}_{a}-P_{\circ}$ die Gleichung:

$$
Q_{a}\left(\frac{1}{a}-\frac{1}{A}\right)=P_{\circ}=\frac{m \beta^{2} c^{2}}{2 e}
$$

Ferner folgt aus der für den Fall eines Teilchens im Felde der Ladung $Q_{a}$ geltenden Differentialgleichung: $m \frac{d^{2} r}{d t^{2}}=-e \frac{Q_{a}}{r^{2}}$ und aus der Anfangsbedingung, dass $r=A$ und $\frac{d r}{d t}=0$ für $t=0$, nach Integration die Gleichung:

und hieraus für die Falldauer $T$ :

$$
\frac{r}{A} \sqrt{\frac{A}{r}-1}+\arctan \sqrt{\frac{A}{r}-1}=\sqrt{\frac{2 Q_{a} e}{A^{3} m}} \cdot t
$$

$$
T=\sqrt{\frac{A^{3} m}{2 Q_{a} e}}\left[\frac{a}{A} \sqrt{\frac{A}{a}-1}+\arctan \sqrt{\frac{A}{a}-1}\right]
$$

oder unter Berücksichtigung der Gleichung (3):

$$
T=\frac{A}{\beta_{\circ} c} \sqrt{\frac{A}{a}-1}\left[\frac{a}{A} \sqrt{\frac{A}{a}-1}+\arctan \sqrt{\frac{A}{a}-1}\right]=\frac{A}{\beta_{\circ} c} \cdot \mathrm{f}\left(\frac{A}{a}\right)
$$

Numerische Werte der hier auftretenden Funktion $\mathrm{f}\left(\frac{A}{a}\right)$ gibt folgende Tabelle:

\begin{tabular}{c|c|c|c|c|c|c|c|c|c|c}
\hline$\frac{A}{a}$ & 1 & 1.25 & 2 & 5 & 10 & 50 & 101 & 401 & 901 & 10001 \\
\hline $\mathrm{f}\left(\frac{\mathrm{A}}{\mathrm{a}}\right)$ & 0 & 0.432 & 1.285 & 3.02 & 4.65 & 11.0 & 15.7 & 31.4 & 47.2 & 158 \\
\hline
\end{tabular}

Vermöge der Gleichungen (1) und (2) ist nun $Q_{a}=2 T I+$ $P_{\circ} a$, andererseits nach Gleichung (3) $Q_{a}=P_{\circ} a \frac{A}{A-a}$, also

$$
2 I T=P_{\circ} a \frac{a}{A-a}
$$

Es kann somit aus (4b) und (5) zu einem vorgegebenen Werte $A$ berechnet werden, welche Emission $I$ dazu nötig ist; die Formel (5) gilt nur, wenn $A$ gross gegen $a$, da nur dann die Voraussetzung erfüllt ist, dass $-Q^{\prime}$ vernachlässigt werden kann.

Ein numerisches Beispiel sei das folgende: eine Kugel von der Grösse des Mondes $\left(a=1.74 \times 10^{8} \mathrm{~cm}, 4 \pi \mathrm{a}^{2}=38 \times 10^{16} \mathrm{~cm}^{2}\right)$ sende $\beta$-Strahlen mit der Anfangsgeschwindigkeit $\beta_{0}=0.3$, Strahlpo- 
tential $P_{0}=82$ stat. Einh. aus. Wenn die Umkehrentfernung $A=401 a$ sein soll (also ungefähr doppelt so gross wie die Distanz Mond-Erde), so berechnet sich nach $(4 b)$ und obiger Tabelle die Steigdauer $T=\frac{6.98 \times 10^{10}}{0.9 \times 10^{10}} \times 31.4=245 \mathrm{sec}$ und daraus weiter nach (5) $I=0.72 \times 10^{5} \frac{\text { stat.-Einh. }}{\mathrm{sec}}=1.5 \times 10^{14} \frac{\text { Elem. quanten }}{\mathrm{sec}}$, bezw. die Zahl $z=4 \times 10-4 \frac{\beta \text {-Strahlen }}{\mathrm{cm}^{2} . \mathrm{sec}}$. Für $A=101 a$, also rund die halbe Distanz Mond-Erde, wird analog $T=30.5 \mathrm{sec}$ und $I=0.49 \times 10^{16}$ $\frac{\text { Elem. quanten }}{\mathrm{sec}}$ oder $z=130 \times 10^{-4} \frac{\beta-\text { Strahlen }}{\mathrm{cm}^{2} \mathrm{sec}}$.

Je höher $I$, bezw. $z$ wird, um so höher ist $Q_{a}$, um so kleiner $A$ und $T$. Schon bei ganz schwacher $\beta$-Strahlung der Mondoberfläche $\left(0.01 \frac{\beta \text { Str. }}{\mathrm{cm}^{2} \mathrm{sec}}\right)$ würden die Teilchen die Erde nicht mehr erreichen, wobei der Mond als absolut atmosphärenlos angenommen ist. Zum Vergleich sei bemerkt, dass eine Oberfläche von der Beschaffenheit der Erdrinde etwa $7 \times 10^{-3} \beta$-Strahlen pro $\mathrm{cm}^{2}$ und sec aussendet.

Bei schnellen Strahlen wird allerdings die einer vorgegebenen Entfernung $A$ zugeordnete Steigdauer verkleinert, $P_{0}$ vergrössert, also $I$ beträch tlich vergrössert. Es lässt sich aber leicht berechnen, dass selbst bei einer enorm raschen (experimentell nicht bekannten) $\beta$-Strahlung mit $\beta_{0}=0.999, P_{0}=35500$ stat. Einh. die zu $A=$ $101 a$ gehörige Emission auf das rund 20000 fache des vorigen Beispieles erhöht würde und somit auch in diesem Falle eine $\beta$ Strahlung von $200 \frac{\text { Strahlen }}{\mathrm{cm}^{2} \mathrm{sec}}$, was bei radioaktiven Messungen als eine zwar gut messbare, aber immerhin schwache Strahlung bezeichnet würde, nicht mehr die Erde erreichen würde.

Für eine fiktive $\alpha$-Strahlung mit $\beta_{0}=0.1, P_{0}=31000$ ergibt sich durch analoge Rechnung, dass zu $A=101 a$ eine Emission von $z=\frac{a-S t r a h l e n}{\mathrm{~cm}^{2} \mathrm{sec}}$ zugeordnct ist.

Zusammenfassend kann man also sagen, dass bei Körpern von den Dimensionen der Weltkörper schon eine für unsere Beobachtungsmethoden schwache oder höchtens mässige Emission korpuskularer Strahlen durch Aufladung des emittierenden Körpers ein bremsendes elektrisches Feld erzeugt, das die Teilchen schon in relativ geringer Entfernung zur Umkehr zwingt und nicht bis zu benachbarten Weltkörpern kommen lässt. Der emittierende Körper nimmt dabei im stationären Zustande eine Ladung an, die grösser als $P_{0} a$ ist. Mit wachsender Stärke der Emission steigt diese Ladung, während die Umkehrentfernung abnimmt. 
4. DIE SELBSTAUFLADUNG BEI GLEICHZEITIGER SPONTANER EMISSION POSITIVER UND NEGATIVER STRAHLUNG.

Wie früher sei eine Kugel (Radius $a$ ) gegeben, die sich in einem Vakuum befindet und gleichzeitig eine positive Strahlung der Gesamtintensität $I_{1}$ und eine negative $I_{2}$ aussende, wobei $I_{2}>I_{1}$, so dass die Kugel eine positive Ladung annimt. $P_{0}$ sei wieder das Strahlpotential der negativen Strahlung.

Unter der (physikalisch nicht realisierbaren) Voraussetzung, dass die negative Strahlung absolut homogen sei und die Oberfläche genau senkrecht verlasse, existiert dann überhaupt kein stationärer Ladungszustand. Denn ist $Q_{a} \leqq P_{0} a$, so gehen alle Teilchen ins Unendliche und infolge des Ueberwiegens der negativer Emission wächst $Q_{a}$ über den Betrag $P_{0} a$ an. Ist aber $Q_{a}>P_{0} a$, so gelangen schliesslich alle emittierten negativen Teilchen wieder zurück, während die fortgehende positive Strahlung $I_{1}$ eine Abnehme von $Q_{a}$ unter $P_{\mathrm{o}} a$ bewirkt. Das Resultat wäre ein periodisches Schwanken um den Betrag $P_{\mathrm{o}} a$.

Nehmen wir aber an, dass die Anfangsgeschwindigkeiten, bezw. Strahlpotentiale der negativen Teilchen nicht absolut gleich, sondern über ein beliebig kleines Intervall $P_{0}$ bis $P_{0}+\triangle P_{0}$ verteilt sind, so lässt sich stets ein dazwischen liegenden Wert $\bar{P}$ angegeben, derart dass die Emission aller Teilchen, deren $P>\bar{P}$ ist, den Betrag $I_{1}$ annimt und somit die positive Emission kompensiert. $P a$ ist dann die stationäre Ladung der Kugel.

\section{DIE SELBSTAUFLADUNG EINES SPONTAN STRAHLENDEN IONI- SIERTEN KöRPERS.}

Im Gegensatze zu den Voraussetzungen der vorigen Abschnitte sei angenommen, dass der emittierende Körper in seiner Oberflächenschichte eine sehr grosse Zahl freier Elektrizitätsträger (Ionen) enthalte, z. B. ein glühender Gasball wie die Sonne oder ein fester Körper mit stark ionisierter Atmosphäre wie die Erde sei.

Wie nehmen ferner wieder eine spontane, negative, senkrecht austretende homogene Korpuskularstrahlung vom Strahlpotential $P_{0}$ und der Gesamtstärke $I$ an. Das von der Selbstaufladung erzeugte Feld treibt die in der Oberfläche ruhenden positiven Ionen nach aussen und es tritt ein stationärer Zustand ein, sobald der Ionenstrom die spontane Emission kompensiert. Die Berechnung des dazu nötigen Feldes erfordert die Lösung eines Problems, das man als Ermittlung des "Raumladungsgrenzstromes im Vakuum 
zwischen konzentrischen Kugelfächen" bezeichnen kann. Analoge Probleme, die sich aber auf den Grenzstrom zwischen parallelen ebenen Platten oder zwischen konzentrischen Zylinderflächen beziehen, wurden von Langmuir ${ }^{7}$ und von Schottky gelöst.

Es werde also zunächst die folgende Aufgabe behandelt: $\mathrm{Ge}-$ geben ist eine Kugel mit stark ionisierter Oberfläche und dem Radius $a$ innerhalb einer leitenden konzentrischen Hohlkugel mit dem Radius $A$; zwischen beiden sei Vakuum. Wenn die innere Kugel auf dem Potential Null, die äussere auf dem konstanten Potentiale $-V_{A}$ gehalten wird, geht ein strom positiver Ionen von innen nach aussen, dessen Stärke $I$ zu berechnen ist.

Unter der Voraussetzung, dass die Endgeschwindigkeit der positiven Ionen (Masse $m$, Ladung $e$ ) nach Durchlaufen der Potentialdifferenz $V_{A}$ immer noch klein gegen die Lichtgeschwindigkeit sei, gilt nach Langmuir (l.c.):

a. Zwischen ebenen Platten in $\operatorname{der}$ Distanz $A$ ist bei der Spannung $V_{A}$ die Stromdichte

$$
i=\frac{1}{9 \pi} \sqrt{\frac{2 e}{m}} \cdot \frac{V_{A}^{3}}{A^{2}}
$$

b. Zwischen konzentrischen Zylindern (Radien $a$ und $A$ ) ist der Strom pro Längeneinheit:

$$
j=\frac{2}{9} \sqrt{\frac{2 e}{m}} \cdot \frac{V_{A}^{\frac{3}{2}}}{A \phi\left(\frac{A}{a}\right)}
$$

wobei $\phi$ eine durch eine unendliche Reihe darstellbare Funktion ist, die bei wachsendem Argument sich rasch dem Grenzwert 1 nähert.

c. Schliesslich beweist Langmuir, dass auch bei beliebig gestalteten Elektroden der Gesamtstrom I proportional zu $V_{A}^{*}$ ist.

Es bleibt also für den hier vorliegenden Fall noch die Abhängigkeit des $I$ von $a$ and $A$ zu bestimmen.

In diesem Falle gilt für eine Entfernung $r$, in der das Potential den Wert $-V(r)$ hat

$$
\frac{m v^{2}}{2}=e V(r)
$$

Eine zweite Gleichung erhalten wir aus der Kontinuitätsbedingung, dass das Produkt aus der Ladung einer Kugelschale, die von $r$ bis $r+d r$ reicht, und der dort vorhandenen Ionengeschwindigkeit $v$ den konstanten Wert $I d r$ haben muss. Da nun die Feld-

'I. LANGmuir, Phys. Ren. (2) 2, 450, 1913; Phys. Zeitschr. 15, 348 u. 516, 1914.

'W. SchortKr, Phys. Zeitschr. 15, 526, 1914. 
stärke, durch $\frac{d V}{d r}$, die gesamte innerhalb $r$ befindliche Ladung durch $r^{2} \frac{d V}{d r}$ und somit die Ladung der Kugelschale durch $d\left[r^{2} \frac{d V}{d r}\right]$ gegeben ist, folgt:

$$
v \frac{d}{d r}\left[r^{2} \frac{d V}{d r}\right]=I
$$

und unter Berücksichtigung der Gleichung (6) weiter

$$
\sqrt{V} \frac{d}{d r}\left[r^{2} \frac{d V}{d r}\right]=\sqrt{\frac{m}{2 e}} \cdot I=K
$$

Durch die Substitution von neuen Variablen

$$
\xi=\log \text { nat } \frac{r}{a} \text { und } \psi=V^{3 / 4}
$$

geht die Differentialgleichung ( 8 ) über in

$$
12 \psi \frac{d^{2} \psi}{d \xi^{2}}+4\left(\frac{d \psi}{d \xi}\right)^{2}+12 \psi \frac{d \psi}{d \xi}=9 K
$$

Die Darstellung von $\psi$ durch eine Potenzreihe und zwar unter Berücksichtigung der Anfangsbedingung, dass für $r=a$ oder $\xi=0$; $V=0$ und daher $\psi=0$ ist, in der Form $\psi=a_{1} \xi+a_{2} \xi^{2}+\ldots \ldots$ $=a_{1} \xi\left[1+\frac{a_{2}}{a_{1}} \xi+\frac{a_{3}}{a_{1}} \xi^{2}+\ldots ..\right]$ liefert dann aus Gleichung für die Werte der Koeffizienten:

$$
a_{1}=\frac{8}{2} \sqrt{K} ; a_{2}=-\frac{8}{10} a_{1} ; a_{8}=\frac{9}{50} a_{1} ; a_{4}=-\frac{{ }_{1}^{-8} \frac{8}{80}}{a_{1}} ; \ldots
$$

und somit die Gleichung

$\psi=V^{3 / 4}=\frac{8}{2} \sqrt{K} \log$ nat $\frac{r}{a}\left[1-\frac{3}{10} \log +\frac{1}{10} 8 \log ^{2}-\frac{8}{10} 80 \log ^{3}+\ldots\right]$ oder ${ }^{*}$

$V_{A}=\left(\frac{3}{2}\right)^{\frac{4}{3}}\left(\frac{m}{2 e}\right){ }^{\frac{1}{3}} I^{\frac{2}{8}}\left(\log \text { nat } \frac{A}{a}\right)^{\frac{4}{8}}\left[1-\frac{3}{10} \log _{0}+\ldots \ldots\right]^{\frac{4}{8}}$

beziehungsweise:

$$
I=\sqrt[4]{\frac{2 e}{m}} \frac{V_{A}}{\left(\log \operatorname{nat} \frac{A}{a}\right)^{2}\left[1-\frac{3}{10} \log +\ldots \ldots\right]^{2}}
$$

Bei wachsender Entfernung der Elektroden steigt also die zur Erziehung eines gegebenen Stromes $I$ notwendige Spannung $V_{A}$ ebenso wie bei den von Langmuir behandelten Fällen ins Unendliche und zwar hier logarithmisch mit $A$. Von einer geladenen ionisierten Kugel wäre also bei endlicher Spannung überhaupt kein endlicher stationärer Strom in den umgebenden unendlichen Raum zu erzielen, im Gegensatz z. B. zu einer geladenen Kugel in 
einem unendlich ausgedehnten nach dem Ohm'schen Gesetze leitenden Medium. Aber diese Unmöglichkeit beruht auf der Wirkung der Raumladung und fällt weg, wenn in hinreichender Entfernung die positive Raumladung der abströmenden Ionen durch eine negative Raumladung kompensiert wird.

Dies ist der Fall bei dem von uns ursprünglich betrachteten Vorgang, wo die ionisierte Kugel gleichzeitig eine negative Emission $I$ besitzt. Im stationären Zustand tritt dann folgende Feldverteilung ein: die Kugel lädt sich auf ein positives Potential II auf; für $r>a$ nimmt $V(r)$ zunächst langsam, dann rasch, dann nach Ueberschreitung eines Wendepunktes wieder langsamer auf den Wert Null in $r=\infty \mathrm{ab}$; die Raumdichte ist überall positiv und sinkt mit wachsenden $r$ asymptotisch und zwar rascher als $\frac{1}{r^{2}}$ abnehmend auf Null. Die Bedingung hierfür ist, dass für grosse Werte von $r$ die Geschwindigkeiten der spontan emittierten negativen Teilchen und der durch das Feld beschleunigten positiven Ionen gleich werden, da sowohl die Raumdichten als auch die Stromdichten (Produkte aus Raumdichte und Geschwindigkeit) gleich sein sollen.

Nun ist in grosser Entfernung für die spontane negative Strahlung mit dem ursprünglichen Strahlpotential $P_{0}$ infolge der Verzögerung $P_{\infty}=P_{0}-$ II, während die feldgetriebenen positiven Ionen schliesslich ein Strahlpotential $I$ erhalten.

Beziehen wir daher den Index 1 auf die spontan emittierten Teilchen, den Index 2 auf die feldgetriebenen Ionen, so gilt nach den im Abschnitt (2) angeführten Beziehungen:

$P_{\circ}-\Pi=\frac{m_{1} c^{2}}{e_{1}}\left(\eta_{1}-1\right)$ und $\Pi=\frac{m_{2} c^{2}}{e_{2}}\left(\eta_{2}-1\right)$ und somit, da für $r=\infty: \beta_{1}=\beta_{2}$ und daher auch $\eta_{1}=\eta_{2}$, die Gleichung

$$
\frac{P_{\circ}-\Pi}{\Pi}=\frac{m_{1} e_{2}}{m_{2} e_{1}} \text { oder } \Pi=P_{\circ} \frac{m_{2} e_{1}}{m_{3} e_{1}+m_{1} e_{2}}
$$

Diese Gleichung (11) muss auch dann erfüllt sein, wenn die anfänglich-zur Berechnung von (10)—vorausgesetzte Bedingung, dass die Endgeschwindigkeit $\beta_{2} c$ für $r=\infty$ klein gegen die Lichtgeschwindigkeit sei, nicht mehr erfüllt ist. Nur die Feldverteilung in der Umgebung der emittierenden Kugel wird dann verändert, derart dass das Gebiet merklicher Raumdichte sich weiter hinaus erstreckt. 
Einsetzen numerischer Werte zeigt, dass bei spontaner Elektronenstrahlung ( $\beta$-Strahlung) und $H$-Ionen als Trägern der feldgetriebenen Strahlung $e_{2}=e_{1}, m_{2}=1848 m_{1}$, also $\Pi=1 \frac{84}{84} P_{\circ}$ wird. Umgekehrt wäre bei spontaner $H$-Strahlung und feldgetriebener Kathoden- (Elektronen-) Strahlung dann $I I=\frac{1}{1849} P_{\mathrm{o}}$ und analog bei spontaner $a$-Strahlung $\mathrm{\Pi}={ }_{3 \mathrm{6} 6 \mathrm{~g}} P_{\text {o }}$.

Bei $A$ nwendung auf die. Sonne ergibt sich daraus das bemerkenswerte Resultat: ob man nun von der Birkeland-Störmer'schen Hypothese einer spontanen Elektronen-emission der Sonne ausgeht oder, der ursprünglichen Auffassung Vegard's folgend, eine spontane positive Strahlung annimmt, in jedem Falle müssen im stationären Zustand in grosser Entfernung beide Strahlengattungen vorhanden sein und zwar mit gleicher mittlerer Intensität und mit gleicher Teilchengeschwindigkeit, so dass entsprechend der grösseren Masse die positive Strahlung eine grössere magnetische Steifigkeit und Durchdringungsfähigkeit besitzt als die negative.

Bekanntlich wurde in mässigen Breiten (Deutschland, England) häufig-ohne jeden Zusammenhang mit ausgesprochenen Nordlichterscheinungen oder magnetischen Störungen-mittels hinreichend lichtstarker Spektroskope die Nordlichtlinie im Lichte des nächtlichen Himmels beobachtet (Wiechert, Rayleigh) und daraus manchmal auf ein allerdings schwaches aber permanentes Nordlicht viel grösserer räumlicher Verbreitung geschlossen. Bei der gewöhnlichen Auffassung ist es eigentlich nicht recht verständlich, warum die magnetisch steife, aber schwache Strahlung so häufig, dagegen die stark ablenkbare intensive Strahlung, welche die Nordlichter im gewöhnlichen Sinne des Wortes erzeugt, seltener ist. Diese Tatsache wird verständlich, wenn wir annehmen, dass fast immer von irgend welchen "aktiven" Stellen der Sonne Bündel spontaner negativer Strahlung ausgehen, aber nur bei günstiger Konstellation die Erde treffen und dann infolge ihrer geringen Steifigkeit hauptsächlich in den Polargegenden intensive Lichterscheinungen hervorrufen; dass aber die feldgetriebene positive Strahlung (in erster Linie wohl $H$-Kerne) gleichmässig, also mit geringerer Stromdichte, von der ganzen Sonnenoberfläche emittiert wird, daher immer zur Erde gelangt und dann bei ihrer grösseren Steifigkeit schwache, jedoch bis in niedere Breiten reichende Lichterscheinungen in der Atmosphäre erzeugt. Auch als Erreger einer sekundären $\gamma$-Strahlung (sehr durchdringenden Strahlung in der Atmosphäre) käme sie eventuell in Betracht.

Ferner ist zu berücksichtigen, dass von der Sonne ausgehende 
Bündel spontaner Strahlen unter günstigen Umständen im Magnetfelde eines Planeten abgelenkt und dann durch die elektrostatischen Kräfte zur entgegengesetzt geladenen Sonne zurückgetrieben werden können; hierdurch wird die Ladungsbilanz der Sonne gestört und die Emission der feldgetriebenen Strahlen verändert. Die vielfach vermuteten "Reflexwirkungen" der Planeten, die sich in Periodizitäten der Sonnenaktivität äussern, welche mit den Perioden der Planeten übereinstimmen, würden so einer physikalischen Erklärung näher gerückt.

Schliesslich seien noch einige numerische Resultate abgeleitet. Aus den direkt beobachteten Daten schliesst Störmer ${ }^{10}$ bei den häufigsten Nordlichtformen auf eine erregende negative Strahlung, bei der das Produkt $H \rho$ von der Grössenordnung $700 \mathrm{r}$. $\mathrm{cm}$ ist. Entsprechend der Tabelle in Abschnitt 2 folgt daraus abgerundet ${ }_{i}$ ein Wert $\beta=0.4$ und $\mathrm{P}=80$ stat. Einh. Nehmen wir $H$-Kerne als hauptsächliche Träger der feldgetriebenen positiven Strahlung an, so folgt aus $\beta=0.4$ der Wert $\Pi=280000$ stat. Einh. und nach Gleichung (11) $P_{\circ}$ nahezu gleich gross. Aus $P_{\circ}$ berechnet sich, dass die Anfangsgeschwindigkeit der spontanen Strahlen zwischen $\left(1-10^{-4}\right)$ und $\left(1-10^{-5}\right)$ liegt. Für die positiven Strahlen hat bei $\beta=0.4$ das Produkt $H \rho$ den Wert $1.3 \times 10^{\circ} \mathrm{T} . \mathrm{cm}$. Die positive Sonnenladung wird $Q_{a}=\Pi_{a}=$ rund $2 \times 10^{16}$ stat. Einh. $=4 \times 10^{25}$ Elementarquanten $\left(a=7 \times 10^{10} \mathrm{~cm}\right.$ gesetzt).

Man kann noch fragen, in welcher Zeit nach Beginn der Spontanstrahlung der stationäre Zustand praktisch erreicht wird. Ohne Kompensation durch positive-Strahlen wäre die Endladung in der Zeit $\tau=\frac{Q_{a}}{I_{1}}$ erreicht; bei einer der vorhandenen Ladung proportionalen Ionenemission wäre $Q(t)=Q_{a}\left(1-e^{\left.-\frac{t}{\tau}\right)}\right.$, also z. B. $Q(t)=$ $0.95 Q_{a}$ für $t=3 \tau$. Tatsächlich erfolgt die Ionenemission nach Formel $(10 b)$ proportionel zu $V^{\frac{8}{2}}$, bezw. $Q^{\frac{3}{2}}$, ist also kleiner als eben angenommen. Die Zeit, nach welcher $95 \%$ der Endladung. erreicht werden, ist daher grösser als $\tau$, kleiner als $3 \tau$, somit von der Grössenordnung $2 \tau$. Soll dies z. B. in rund 22 Stunden $=80000 \mathrm{sec}$ erreicht werden, so muss $I=\frac{4 \times 10^{25} \text { Elementarquanten }}{4 \times 10^{4} \text { Sekunden }}=10^{21}$ sein. Dem entspricht eine Emission von nur $\frac{1}{60}$ pro $\mathrm{cm}^{2}$ und $\mathrm{sec}$ für die positiven Ionen und von $17 \beta$-Strahlen pro $\mathrm{cm}^{2}$ und sec für die spontane Strahlung, falls 1 Promille der Sonnenoberfläche als "aktiv" angenommen wird. Bei stärkerer Emission erfolgt die Herstellung des stationären Zustandes entsprechend rascher.

Vergl. insbesondere L. A. Bauer, Terr. Mag-, 26, 1921, pp. 40, 65-66, und 113-115.

10C. Störmer, Geofys. Publ. 1, Nr. 5, Kristiania, 1921. 
ZUSAMMENFASSUNG.

1. Es werden kurz die beobachteten Erscheinungen besprochen, die zu Hypothesen über die Existenz von korpuskularen kosmischen Strahlungen geführt haben, und die Wichtigkeit bctont, die theoretischen Konsequenzen bezüglich des Ladungssustandes der emittierenden Weltkörper abzuleiten

2. Die Bestimmungsmerkmale korpuskularer Strahlen werden besprochen, speziell das "Strahlpotential" P, das als Quotient der kinetischen Energie und der elektrischen Ladung eines Teilchens definiert ist, sowie die magnetische Ablenkbarkeit. Eine Tabelle zur Berechnung dieser Grössen bei verschiedenen Geschwindigkeiten ist beigefügt.

3. Die stationäre Ladung einer Kugel, die spontan Korpuskularstrahlen ins umgebende Vakuum aussendet, wird berechnet. Sie ist grösser als das Produkt $P a$ aus Strahlpotential $P$ und Kugelradius $a$ und steigt mit der Intensität der Emission an. Die emittierten Teilchen werden dabei in einer Entfernung $A$ zur Umkehr gebracht; der Wert von $A$ wird ebenfalls berechnet. Numerische Beispiele für den Mond als supponierte Strahlenquelle werden beigefügt.

4. Die stationäre Ladung bei gleichzeitiger spontaner Emission von positiven und negativen Korpuskularstrahlen wird berechnet.

5. Analoge Rechnungen werden durchgeführt unter der Annahme, dass eine in der Oberfläche stark ionisierte Kugel eine spontane Strahlung und daher infolge ihrer Aufladung eine kompensierende feldgetriebene Ionenstrahlung aussende. Im stationären Zustande sind in grosser Entfernung dann positive und negative Strahlen von gleicher mittlerer Intensität und gleicher Geschwindigkeit vorhanden.

Die Resultate werden auf die Verhältnisse bei der Sonne angewendet und einige numerische Beispiele berechnet.

Physikalisches Institut Der UnIVersität INNSBRUCK. 